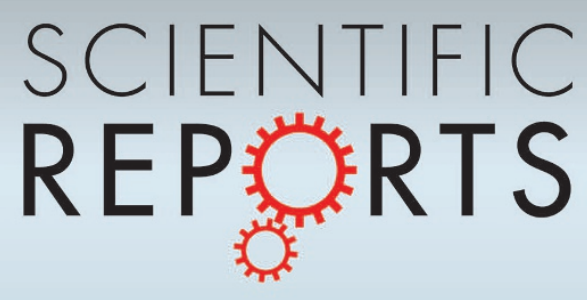

\title{
OPEN Interlayer interactions in graphites
}

SUBJECT AREAS:

CONDENSED-MATTER

PHYSICS

THEORY AND COMPUTATION

Received

28 August 2013

Accepted

8 October 2013

Published

6 November 2013

Correspondence and requests for materials should be addressed to

N.-X.C. (nanxian@

tsinghua.edu.cn)

\author{
Xiaobin Chen ${ }^{1}$, Fuyang Tian ${ }^{2,3}$, Clas Persson ${ }^{3,4}$, Wenhui Duan' \& Nan-xian Chen ${ }^{1,2}$
}

'Department of Physics and State Key Laboratory of Low-Dimensional Quantum Physics, Tsinghua University, Beijing 100084, China, ${ }^{2}$ Institute for Applied Physics, University of Science and Technology Beijing, Beijing 100083, China, ${ }^{3}$ Department of Materials Science and Engineering, Royal Institute of Technology, 10044 Stockholm, Sweden, ${ }^{4}$ Department of Physics, University of Oslo, 0316 Oslo Norway.

Based on $a b$ initio calculations of both the $\mathrm{ABC}$ - and $\mathrm{AB}$-stacked graphites, interlayer potentials (i.e., graphene-graphene interaction) are obtained as a function of the interlayer spacing using a modified Möbius inversion method, and are used to calculate basic physical properties of graphite. Excellent consistency is observed between the calculated and experimental phonon dispersions of AB-stacked graphite, showing the validity of the interlayer potentials. More importantly, layer-related properties for nonideal structures (e.g., the exfoliation energy, cleave energy, stacking fault energy, surface energy, etc.) can be easily predicted from the interlayer potentials, which promise to be extremely efficient and helpful in studying van der Waals structures.

T he interlayer cohesive properties in graphite have been an attractive issue for more than 50 years ${ }^{1-9}$, and revive these years due to the observation of graphene and few-layer graphite ${ }^{10-12}$. Although there exist $A B$ and $\mathrm{ABC}$ stackings of graphite in nature, a stable single crystal specimen can be well prepared only for $\mathrm{AB}-$ stacked graphite ${ }^{13-15}$. This fact has greatly hindered the research on layer-related properties of graphite and as a result, the overwhelming majority of theoretical efforts have been devoted only to AB-stacked graphite e $^{5-7,9}$. Actually, it is a physical challenge to theoretically describe the van der Waals (vdW) interactions in complex materials, and extensive work is still needed for better understanding the interlayer cohesion in graphite both theoretically and experimentally. However, as will be shown in this work, the interlayer binding energies in graphites with various stackings are closely related. Therefore, instead of focusing on only one type of stackings, the present work combines both $\mathrm{AB}$ - and $\mathrm{ABC}$-stacked graphites to study interlayer potentials in graphite or graphene-graphene interactions, which can be extracted by combining $a b$ initio calculations with Chen-Möbius inversion ${ }^{16-18}$, and further be employed to study layer-related properties of graphite systems over a wide range.

In this work, we use ab initio calculations to generate binding energies of $\mathrm{ABC}$ - and $\mathrm{AB}$-stacked graphite as functions of the layer separation. Afterward, the graphene-graphene interaction potentials are obtained using Möbius inversion procedure. Finally, a few examples are given to show the validity and usefulness of the deduced interlayer potentials. These potentials are expected to be powerful in studying graphites with arbitrary $c$-axis stacking order ${ }^{19}$ or layer spacings, which can be too large for $a b$ initio calculations, and in understanding even predicting the transformation of stacking orders in multi-layer graphen $e^{20}$. Further by including the variance of lattice parameters, this method can be extended to study strain effects on stacking orders ${ }^{21}$.

\section{Results}

By evaluating values of the equilibrium interlayer distance, $d_{0}$, the binding energy, $E_{b}$, and the elastic coefficient, $\mathrm{C}_{33}$, and making comparison with experimental values as listed in Table I, we find that PBE/DFT-D2 is the best exchange-correlation functional among the functionals that we choose for presenting the ab initio binding energies of graphites. As a verification, we check the asymptotic behavior of interlayer binding energy of ABstacked graphene. In Fig. 1, we plot the interlayer binding energy of AB-stacked graphite and a fitting by the function $y=\mathrm{A} / d^{4.2}+\mathrm{C}$ as a function of the interlayer distance, $d$. Obviously, the interlayer binding energy curve from PBE/DFT-D2 between $4 \sim 8 \AA$ satisfies the rule very well, which agrees with previous finding using random phase approximation in $\mathrm{AB}$-stacked graphite ${ }^{6,22}$. What is more, we note that the PBE/DFT-D2 calculated $E_{b}$ of $\mathrm{AB}$-stacked graphite is greater than that of $\mathrm{ABC}$-stacked one at equilibrium interlayer distance by $0.25 \mathrm{meV} /$ atom, which qualitatively agrees with the estimation of its natural abundance $(80 \%)^{23}$.

Despite of four different arrangements along hexagonal close packed (hcp) $\langle 0001\rangle$ direction, AB, ABC, ABAC, and AA stackings, there are only two different graphene-graphene interaction potentials due to the three-fold rotation symmetry in individual graphene layers. Herein, we use $\phi^{\mathrm{AB}}(d)$ to denote the interlayer potentials between $\mathrm{A}-\mathrm{B}, \mathrm{A}-\mathrm{C}$, or $\mathrm{B}-\mathrm{C}$ layers, and $\phi^{\mathrm{AA}}(d)$ those between aligned layers, i.e., $\mathrm{A}-\mathrm{A}, \mathrm{B}-\mathrm{B}$, and $\mathrm{C}-\mathrm{C}$ layers. 
Table I | Ab initio equilibrium interlayer distance $d_{0}$ (in $\AA$ ), binding energy $E_{b}$ (in meV/atom), and elastic constant $C_{33}$ (in $G P a$ ) obtained using various exchange-correlation functionals for $A A-, A B$-, and $A B C$-stacked graphites

\begin{tabular}{|c|c|c|c|c|c|c|c|c|c|}
\hline \multirow[b]{2}{*}{ Method } & \multicolumn{3}{|c|}{$A B-$} & \multicolumn{3}{|c|}{$A B C-$} & \multicolumn{3}{|c|}{ AA- } \\
\hline & $d_{0}$ & $E_{b}$ & $C_{33}$ & $d_{0}$ & $E_{b}$ & $C_{33}$ & $d_{0}$ & $E_{b}$ & $C_{33}$ \\
\hline $\begin{array}{l}\text { LDA(DFT) } \\
\text { LDA/DFT-D2 } \\
\text { PBE } \\
\text { PBE/DFT-D2 } \\
\text { optPBE/vdW-DF } \\
\text { OptB88/vdW-DF } \\
\text { rPW86/vdW-DF2 } \\
\text { Expts }\end{array}$ & $\begin{array}{l}3.334 \\
2.989 \\
4.419 \\
3.231 \\
3.447 \\
3.356 \\
3.524 \\
3.336^{a}\end{array}$ & $\begin{array}{c}23.67 \\
114.9 \\
0.88 \\
55.15 \\
63.70 \\
69.56 \\
52.08 \\
52 \pm 5^{b}, 31 \pm 2^{h} \\
43^{d}, 35 \pm 10^{f}\end{array}$ & $\begin{array}{l}28.34 \\
95.23 \\
1.390 \\
42.44 \\
33.15 \\
40.37 \\
34.11 \\
40.7^{c} \\
38.7^{e}, 47^{g}\end{array}$ & $\begin{array}{l}3.340 \\
2.989 \\
4.420 \\
3.232 \\
3.450 \\
3.359 \\
3.525\end{array}$ & $\begin{array}{c}23.20 \\
114.1 \\
0.99 \\
54.80 \\
63.63 \\
69.20 \\
52.15\end{array}$ & $\begin{array}{l}27.88 \\
97.59 \\
1.396 \\
41.68 \\
33.14 \\
40.19 \\
33.16\end{array}$ & $\begin{array}{l}3.622 \\
3.198 \\
4.586 \\
3.492 \\
3.625 \\
3.545 \\
3.670\end{array}$ & $\begin{array}{r}14.15 \\
55.53 \\
0.68 \\
41.55 \\
56.41 \\
58.81 \\
45.74\end{array}$ & $\begin{array}{l}19.26 \\
46.29 \\
1.297 \\
30.28 \\
32.46 \\
35.67 \\
32.28\end{array}$ \\
\hline 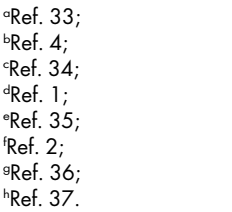 & & & & & & & & & \\
\hline
\end{tabular}

Based on the PBE/DFT-D2 results and using a modified Möbius method as will be introduced in the Method section, interlayer potentials $\phi^{\mathrm{AA} / \mathrm{AB}}(d)$ can be extracted. In Fig. 2, we plot the interlayer potentials $\phi^{\mathrm{AB}}(d)$ and $\phi^{\mathrm{AA}}(d)$ as functions of $d$. As shown in the figure, $\phi^{\mathrm{AB}}(d)$ takes its minimum value at $d_{0}$, and $\phi^{\mathrm{AA}}(d)$ monotonically increases from $d>5 \AA$. (Note that $\phi^{\mathrm{AA}}(d)$ is plotted only for large interlayer distances, since equations (8) and (9) requires that $d \geq 2 d_{1}$, where $d_{1}$ is the minimum interlayer distance we set for $a b$ initio calculation).

So as to better describe the interlayer potentials, we use the Rahaman-Stillinger-Lemberg (RSL2) function

$$
\begin{aligned}
\phi(d)= & D_{0} e^{y\left(1-\frac{d}{R_{0}}\right)}+\frac{a_{1}}{1+e^{b_{1}\left(d-c_{1}\right)}} \\
& +\frac{a_{2}}{1+e^{b_{2}\left(d-c_{2}\right)}}+\frac{a_{3}}{1+e^{b_{3}\left(d-c_{3}\right)}},
\end{aligned}
$$

to fit the deduced interlayer potentials. With the total 12 adjustable parameters, $a_{i}, b_{i}, c_{i},(i=1,2,3)$, this function can well describe pair interactions between layers. The best-fit parameters for the interlayer potentials are listed in Table II.

From the deduced interlayer potentials $\phi^{\mathrm{AB}}(d)$ and $\phi^{\mathrm{AA}}(d)$, we can reproduce the interlayer binding energies of variously stacked graphites as:

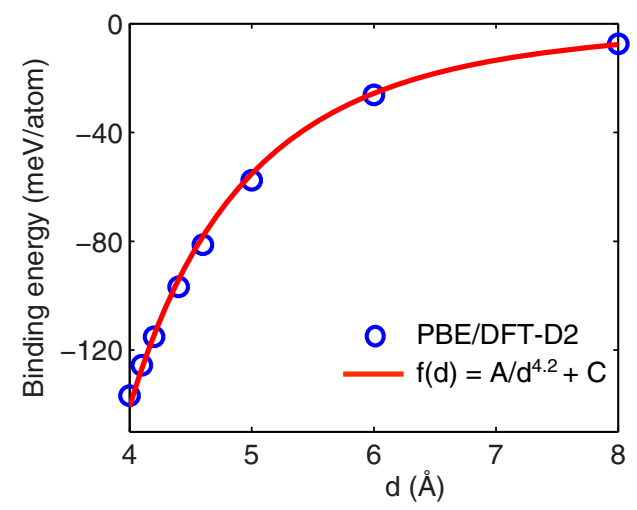

Figure 1 Binding energy of $\mathrm{AB}$-stacked graphite as a function of interlayer distance. The PBE/DFT-D2 results are fitted by the function $y=$ $\mathrm{A} / d^{4.2}+\mathrm{C}$, where $\mathrm{C}$ and $\mathrm{A}$ are coefficients.

$$
\begin{aligned}
E_{\phi}^{\mathrm{AA}}(d)= & \sum_{n=1}^{\infty} \phi^{\mathrm{AA}}(n d), \\
E_{\phi}^{\mathrm{AB}}(d)= & \sum_{n=1}^{\infty} \phi^{\mathrm{AB}}[(2 n-1) d]+\sum_{n=1}^{\infty} \phi^{\mathrm{AA}}(2 n d), \\
E_{\phi}^{\mathrm{ABC}}(d)= & \sum_{n=1}^{\infty} \phi^{\mathrm{AA}}(3 n d)+\sum_{n=1}^{\infty} \phi^{\mathrm{AB}}[(3 n-1) d] \\
& +\sum_{n=1}^{\infty} \phi^{\mathrm{AB}}[(3 n-2) d],
\end{aligned}
$$

where the subscript $\phi$ means that the functions are evaluated from $\phi^{\mathrm{AA} / \mathrm{AB}}(d)$, instead of directly obtained from ab initio calculations. The reconstructed binding energy curves of $\mathrm{AB}$ - and $\mathrm{ABC}$-stacked graphites are plotted and compared in Fig. 3. It is clearly shown in Fig. 3(a) and (b) that the binding energies of AB- and ABC-stacked graphites are well reproduced. Especially, the interlayer binding energy difference between $\mathrm{AB}$ - and $\mathrm{ABC}$-stacked graphites is well distinguished in Fig. 3(c). Also, the obtained binding energy curve of AA-stacked graphite shows good consistency with the $a b$ initio

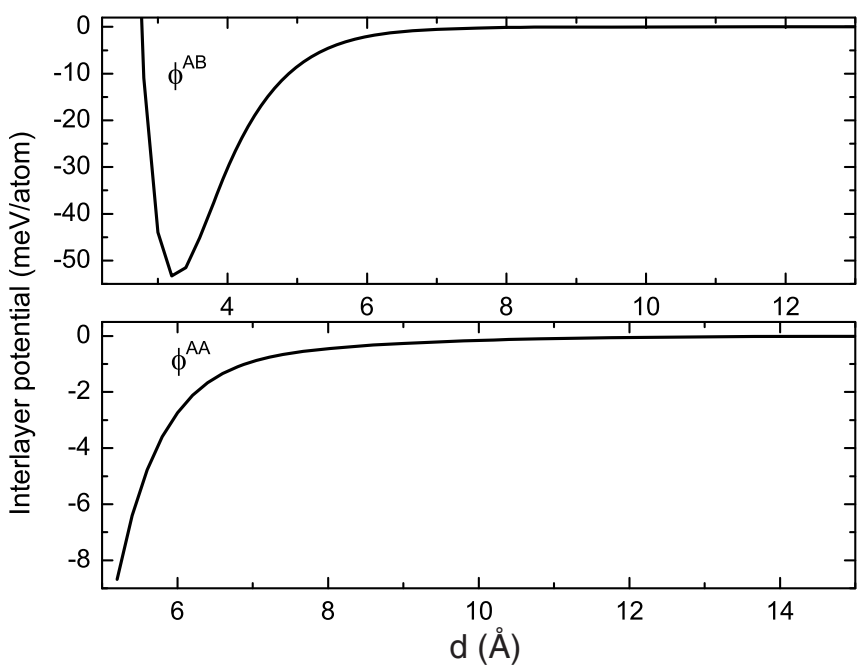

Figure $2 \mid$ Interlayer potentials $\phi^{\mathrm{AB}}(d)$ and $\phi^{\mathrm{AA}}(d)$. 
(a)

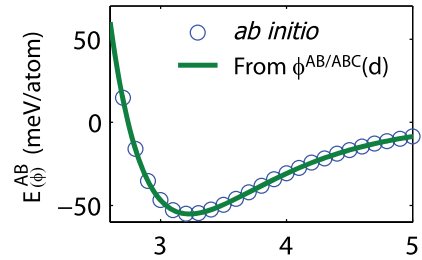

(b)
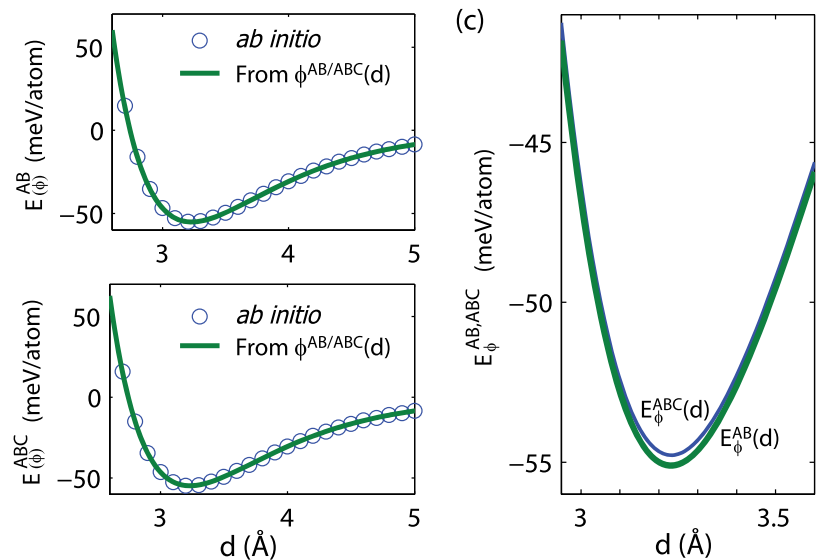

Figure 3 Binding energy curves of (a) AB- and (b) ABC-stacked graphite as functions of interlayer distance. (c) Comparison of binding energies of $\mathrm{AB}$ - and $\mathrm{ABC}$-stacked graphites obtained from interlayer potentials near the equilibrium distance.

results for $d \geq 2 d_{1}$, where $d_{1}$ is the minimum $d$ used in ab initio calculations (data not shown).

\section{Discussion}

Besides giving us more insight into weak interlayer interactions, interlayer potentials are powerful in studying layer-related properties, such as $c$-axis phonon dispersion, exfoliation energy, cleave energy, surface energy, and stacking fault energy.

Since interlayer potentials can fully describe the interactions between layers along the hcp $\langle 0001\rangle$ direction, we can use interlayer potentials to calculate interlayer vibrations. We take a graphene layer as a whole and use the interlayer potentials to describe the graphenegraphene interaction. The phonon dispersion results, which are actually the $c$-axis vibration spectra, are illustrated in Fig. 4, where the branches of phonon dispersion for AB-stacked graphite are well consistent with those of the neutron scattering data from Ref. 24. In unit of $\mathrm{meV}$, the values of frequency are $17 \mathrm{meV}, 1.5 \mathrm{meV}$, and 0 at the $\Gamma$ point from the interlayer potentials, which qualitatively agree with the experimental values: 15,6 , and $0 \mathrm{meV}^{24}$.

Exfoliation energy, $E_{\text {exf }}$, is the energy required to remove one graphene plane from the surface of a bulk graphite, and can be used to estimate $E_{b}$. With the interlayer potentials, we can easily obtain $E_{\text {exf }}$. For instance, the exfoliation energy for $\mathrm{AB}$-stacked graphite is followed as:

$$
E_{\text {exf }}=\sum_{n=1}^{\infty}\left\{\phi^{\mathrm{AB}}\left[(2 n-1) d_{0}\right]+\phi^{\mathrm{AA}}\left(2 n d_{0}\right)\right\} .
$$

Compared to equation (2), the $E_{\text {exf }}$ in the above equation is exactly half of the interlayer binding energy. Thereby, the exfoliation energy is about $55.16 \mathrm{meV} /$ atom. Similarly, we can obtain an exfoliation energy $57.14 \mathrm{meV} /$ atom when two graphene layers are exfoliated.

Another method to calculate $E_{b}$ is to get the cleave energy, $E_{\mathrm{cl}}$, which is the interaction energy between two semi-infinite crystals. In AB-stacked graphite, it shows that

$$
E_{\mathrm{cl}}=\sum_{n=1}^{\infty}\left\{(2 n-1) \phi^{\mathrm{AB}}\left[(2 n-1) d_{0}\right]+2 n \phi^{\mathrm{AA}}\left(2 n d_{0}\right)\right\} .
$$

Apparently, the cleave energy is bigger than the exfoliation energy in AB-stacked graphite. The difference is about $2.3 \mathrm{meV} /$ atom.

The surface energy is defined as the excess free energy per unit area of a particular crystal facet. It is usually calculated using the supercell method: $E_{\text {surf }}=\left(E_{s}-N E_{0}\right) / 2 A$, where $E_{s}$ is the total energy of the supercell, $E_{0}$ the bulk energy per layer, $A$ the surface area of the
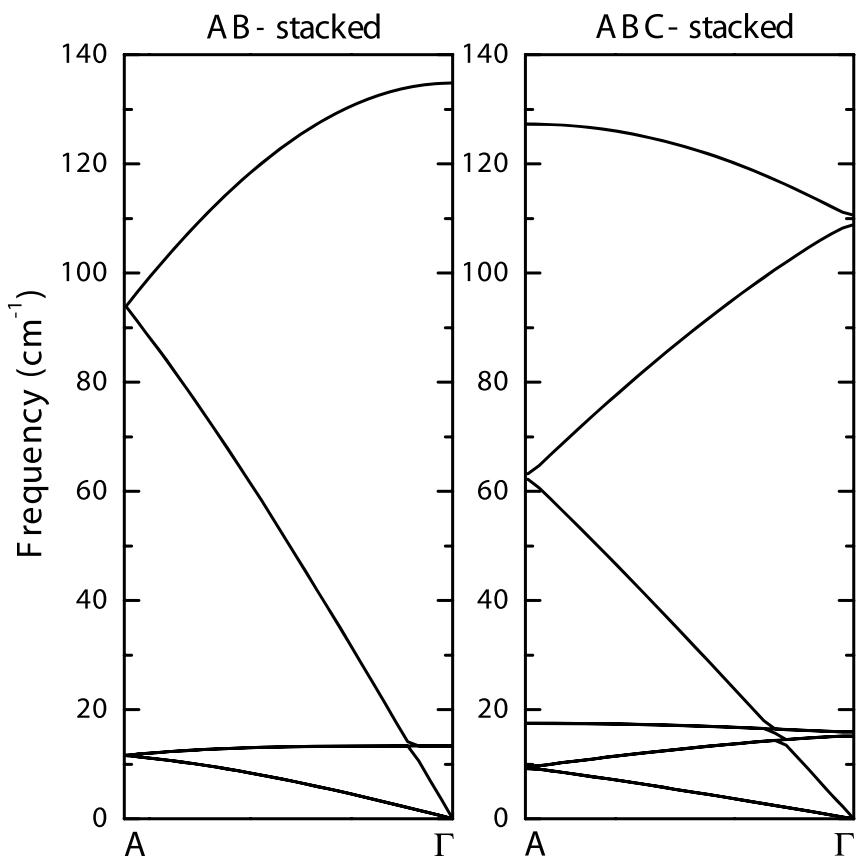

Figure $4 \mid$ Phonon dispersion of $\mathrm{AB}$ - and $\mathrm{ABC}$-stacked graphites along the $c$ axis.

supercell, and the factor 2 derived from two surfaces in the supercell model. $E_{\text {surf }}$ can also be easily computed by interlayer potentials, which yields that the surface energy is about $351 \mathrm{~mJ} / \mathrm{m}^{2}$ for ABstacked graphite. The interlayer potentials may also be used to establish the size of the surface relaxation and the impact on the formation energy. In the present surface relaxation is only between the surface layer and the neighboring layer. The surface relaxation energy from the interlayer potentials is $338 \mathrm{~mJ} / \mathrm{m}^{2}$, which is $13 \mathrm{~mJ} / \mathrm{m}^{2}$ smaller than the ideal surface energy. The relaxation interlayer distance is about $3.238 \AA$, while the equilibrium interlayer distance is $3.231 \AA$. Similarly, we also calculated the surface energy of ABC-stacked graphite along hcp $\langle 0001\rangle$. Its value is $344 \mathrm{~mJ} / \mathrm{m}^{2}$ which is smaller by $7 \mathrm{~mJ} / \mathrm{m}^{2}$ than that of $\mathrm{AB}$-stacked graphite. Note that the the surface energy calculated with LDA is only $75 \mathrm{~mJ} / \mathrm{m}^{2}$ for AB- stacked graphite as reported in Ref. 25. The large difference is mainly derived from the interlayer cohesive energy of the different exchange-correlation approximations.

Also, commonly seen stacking faults can be studied. We calculate the intrinsic stacked fault energies of $\mathrm{AB}$-stacked graphite according to the equation $E_{\text {surf }}=\left(E_{s f}-E_{\text {ideal }}\right) / A_{s f}$. Herein $E_{s f}$ is the energy of the intrinsic stacking fault, $E_{\text {ideal }}$ is the energy of the perfect stacking, and $A_{s f}$ is the area of the stacking fault plane.

Ideal stackings are $\cdots \mathrm{ABAB} \cdots$ for $\mathrm{AB}$-stacked graphite, $\cdots$ ABCABC $\cdots$ for ABC-stacked graphite, $\cdots$ ABAC $\cdots$ for the ABAC-stacked graphite, and $\cdots$ AA $\cdots$ for AA-stacked graphite. Herein, we only consider one stacking fault from $\mathrm{AB}$-stacked to AA-, ABC-, and ABAC-stacked graphites. From ABC- to AB-stacked graphite, namely $\cdots A B C A B C: A B C A B C \cdots$ to $\cdots A B C A B$ : ABCABCA ... the stacking fault energy is $-8.0 \mathrm{~mJ} / \mathrm{m}^{2}$, which also indicates that $\mathrm{AB}$-stacked graphite is more stable than $\mathrm{ABC}$-stacked one. From $\mathrm{AB}$ - to $\mathrm{AA}$-stacked graphite, $\cdots \mathrm{ABAB}: \mathrm{ABAB} \cdots$ to $\cdots$ ABAB:BABA $\cdots$ the stacking fault energy is $148 \mathrm{~mJ} / \mathrm{m}^{2}$. From AB- to ABAC-stacked graphite, $\cdots$ ABA $\vdots$ B ABA $\cdots$ to $\cdots$ ABA $\mathrm{C}: \mathrm{ABA} \cdots$, the stacking fault energy is $7.9 \mathrm{~mJ} / \mathrm{m}^{2}$, which implies that the interlayer binding energy of $\mathrm{ABC}$-stacked graphite is very close to that of ABAC-stacked graphite.

In summary, we have obtained interlayer potentials $\phi^{\mathrm{AB} / \mathrm{AA}}(d)$ combining the Möbius inversion method with $a b$ initio results. The interlayer potentials can well reproduce binding energies of 
Table II | Best-fit parameters for the interlayer potentials using RSL2 function [see equation (1)]

\begin{tabular}{|c|c|c|c|c|c|}
\hline Parameters & $\phi^{\mathrm{AB}}$ & $\phi^{\mathrm{AA}}$ & Parameters & $\phi^{\mathrm{AB}}$ & $\phi^{\mathrm{AA}}$ \\
\hline$D_{0}$ (meV/atom) & -3.975825 & -0.028157 & $a_{2}(\mathrm{meV} /$ atom $)$ & -0.000232 & -0.000005 \\
\hline$R_{0}(\AA)$ & 1.000000 & 1.000000 & $b_{2}\left(\AA^{-1}\right)$ & 12.409694 & 12.409694 \\
\hline$y$ & 1.357722 & 0.501727 & $c_{2}(\AA)$ & 28.113815 & 28.113815 \\
\hline$b_{1}\left(\AA^{-1}\right)$ & 3.245134 & 5.168542 & $b_{3}\left(\AA^{-1}\right)$ & 5.069858 & 1.983866 \\
\hline$c_{1}(\AA)$ & 1.786003 & 0.110757 & $c_{3}(\stackrel{\AA}{A})$ & 3.289036 & 2.950554 \\
\hline
\end{tabular}

graphites with $\mathrm{AB}$ or $\mathrm{ABC}$ stackings, indicating the validity of the interlayer potentials, and can be used to predict many properties such as $c$-axis phonon dispersion, exfoliation energy, surface energy, surface relaxation, and stacking fault energies of various graphites. We conclude that the interlayer potentials offer an easy and efficient way to investigate layer-related properties of graphites, and the combination of Möbius inversion method and $a b$ initio calculation may find wide applications in layered structures.

\section{Methods}

There exist numerous ab initio calculation methods with different exchange-correlation approximations to deal with the vdW interaction. However, it is still difficult to exactly include the nonlocal long-range vdW interaction. In the present work, we have tested seven different exchange-correlation functionals (see Table I $)^{26-29}$. Considering only the interaction between layers, we fix the interatomic distance within each layer and obtain the corresponding total energy at different interlayer distances by changing the interlayer distance. After extracting the sum of energies of individual layers from the total energy, we get the interlayer binding energy as a function of interlayer distance. All ab initio calculations have been performed within the Vienna $A b$ initio Simulation Package (VASP) ${ }^{30,31}$. To take into account the small energy change caused by the weak vdW interaction, the cutoff energy is taken as $900 \mathrm{eV}$, the $\mathrm{k}$ mesh in the first Brillouin zone is set to be $16 \times 16 \times 12$, and the convergence of total energy is $2 \times 10^{-7} \mathrm{eV}$. In order to better describe the graphenegraphene interaction in graphite, we need to select the best exchange-correlation functional for the system for further analysis. As usually done in literature, we use the consistency of calculated results to the experimental values of equilibrium interlayer distance $d_{0}$, the binding energy $E_{b}$, and the elastic coefficient $C_{33}$, as the criteria. The Morse function ${ }^{32}$ is chosen as the state function to fit the binding energy values near the ideal interlayer distance to get $d_{0}, E_{b}$, and $C_{33}$.

In our calculations, the interlayer binding energy is defined as the atom-averaged energy difference between separated layers and bound layers as:

$$
E_{b}=-\left(E_{\text {tot }}-N \cdot E_{\text {unit }}\right) /\left(N \times N_{\text {atom }}\right),
$$

where $E_{b}$ represents the interlayer binding energy in unit of $\mathrm{meV} / \mathrm{atom}, E_{\mathrm{tot}}$ is the total energy of N layers in the calculational model, $E_{\text {unit }}$ corresponds to the energy of a single layer, i.e., graphene, and $N_{\text {atom }}$ is the number of atoms per layer. While, the elastic coefficient $C_{33}$, which is along hcp $\langle 0001\rangle$ direction in hexagonal lattice, is defined as:

$$
C_{33}=\frac{\partial(\partial E / S \partial d)}{\partial\left(d / d_{0}\right)}=\frac{d_{0}}{S} \cdot \frac{\partial^{2} E}{\partial d^{2}}
$$

with

$$
S=3 \sqrt{3} \cdot a_{0}^{2} / 4,
$$

where $d_{0}$ is the equilibrium interlayer distance, and $a_{0}=1.42 \AA$ is the interatomic distance within a layer.

Given the $a b$ initio results of binding energy functions for AB- and ABC-stacked graphites, we can use the Möbius inversion method to generate interlayer potentials, or graphene-graphene interaction potentials.

In the following, we use Möbius inversion method to get the interlayer potentials from binding energies of $\mathrm{AB}$ - and $\mathrm{ABC}$-stacked graphites.

For $\mathrm{AB}$-stacked graphite, the interlayer binding energy $E^{\mathrm{AB}}(d)$ is given as

$$
E^{\mathrm{AB}}(d)=\sum_{n=1}^{\infty} \phi^{\mathrm{AB}}[(2 n-1) d]+\sum_{n=1}^{\infty} \phi^{\mathrm{AA}}(2 n d) .
$$

For ABC-stacked graphite, the binding energy can be expressed similarly as

$$
E^{\mathrm{ABC}}(d)=\sum_{n=1}^{\infty} \phi^{\mathrm{AA}}(3 n d)+\sum_{n=1}^{\infty} \phi^{\mathrm{AB}}[(3 n-1) d]+\sum_{n=1}^{\infty} \phi^{\mathrm{AB}}[(3 n-2) d] .
$$

Assuming that $E^{\mathrm{ABC}}(d)$ and $E^{\mathrm{AB}}(d)$ are known, we can combine the equations (8) and (9) to determine $\phi^{\mathrm{AB}}(d)$ and $\phi^{\mathrm{AA}}(d)$. Introducing the energy difference $\Delta^{\mathrm{AB}}(d)$ as

$$
\Delta^{\mathrm{AB}}(d)=E^{\mathrm{ABC}}(d)-E^{\mathrm{AB}}\left(\frac{3}{2} d\right),
$$

we obtain the following formula:

$$
\begin{aligned}
\Delta^{\mathrm{AB}}(d)= & \sum_{n=1}^{\infty} \phi^{\mathrm{AB}}[(3 n-1) d]+\sum_{n=1}^{\infty} \phi^{\mathrm{AB}}[(3 n-2) d] \\
& -\sum_{n=1}^{\infty} \phi^{\mathrm{AB}}\left[\frac{3}{2}(2 n-1) d\right]
\end{aligned}
$$

This equation can be written in a simple form:

$$
\Delta^{\mathrm{AB}}(d)=\sum_{n=1}^{\infty} r(n) \phi^{\mathrm{AB}}[b(n) d]
$$

by introducing $(n=1,2, \cdots)$

$$
\begin{aligned}
& r(n)= \begin{cases}-1, & \text { if } n=3 k-1, \\
1, & \text { otherwise },\end{cases} \\
& b(n)= \begin{cases}3 k-2, & \text { if } n=3 k-2, \\
3 k-3 / 2, & \text { if } n=3 k-1, \\
3 k-1, & \text { otherwise }\end{cases}
\end{aligned}
$$

The idea of solution is simply to work with the multiplicative closure $B=\{B(n)\}_{n=1}^{\infty}$ of the original set $b=\{b(n)\}_{n=1}^{\infty}$, i.e., $B$ is a multiplicative semi-group containing the elements of $b$, and all products of them ${ }^{16}$. Correspondingly, the set $\{r(n)\}_{n=1}^{\infty}$ is extended to another set $\{R(n)\}_{n=1}^{\infty}$ as

$$
\Delta^{\mathrm{AB}}(d)=\sum_{n=1}^{\infty} R(n) \phi^{\mathrm{AB}}[B(n) d],
$$

where $R(n)$ satisfies

$$
R(n)= \begin{cases}r\left\{b^{-1}[B(n)]\right\}, & \text { if } B(n) \in b(n), \\ 0, & \text { otherwise. }\end{cases}
$$

In the above notation, $b^{-1}$ refers to the inverse of the arithmetic function $b(n)$, i.e., a mapping from the set $\{b(n)\}_{n=1}^{\infty}$ back to the natural numbers. Similarly to the traditional Möbius inversion method, we can write down a symmetric form as a solution of $\phi^{\mathrm{AB}}(d)$ :

$$
\phi^{\mathrm{AB}}(d)=\sum_{n=1}^{\infty} J(n) \Delta^{\mathrm{AB}}[B(n) d],
$$

and substitute it into equation (14) to determine $J(n)$ :

$$
\sum_{B(n) \mid B(k)} J(n) R\left\{B^{-1}\left[\frac{B(k)}{B(n)}\right]\right\}=\delta_{k, 1},
$$

from which $J(n)$ can be obtained recursively.

In order to solve $\phi^{\mathrm{AA}}(d)$, we define

$$
\Delta^{\mathrm{AA}}(d)=E^{\mathrm{AB}}\left(\frac{d}{2}\right)-\sum_{n=1}^{\infty} \phi^{\mathrm{AB}}\left[\left(n-\frac{1}{2}\right) d\right],
$$

and then from equation (8), we have

$$
\Delta^{\mathrm{AA}}(d)=\sum_{n=1}^{\infty} \phi^{\mathrm{AA}}(n d)
$$

Then, by virtue of Möbius theorem, $\phi^{\mathrm{AA}}(d)$ can be obtained as:

$$
\phi^{\mathrm{AA}}(d)=\sum_{n=1}^{\infty} \mu(n) \Delta^{\mathrm{AA}}(n d),
$$


where $\mu(n)$ is the Möbius function defined as:

$$
\mu(n)= \begin{cases}1, & \text { if } n=1, \\ (-1)^{s}, & \text { if } n \text { is a product of } s \text { distinct primes, } \\ 0, & \text { otherwise. }\end{cases}
$$

Apparently, the interlayer interactions can be strictly considered up to arbitrarydistance neighboring layers on the bases of the above Chen-Möbius inversion method. According to equations (16) and (19), the premise condition of getting $\phi^{\mathrm{AB}}(d)$ and $\phi^{\mathrm{AA}}(d)$ is to obtain the interlayer binding energies, $E^{\mathrm{ABC}}(d)$ and $E^{\mathrm{AB}}(d)$ Note that as a consequence, the reliability of interlayer potentials depends on the accuracy of interlayer binding energies, and besides $a b$ initio calculations, experimental binding energy curves, can also be used to generate interlayer potentials.

1. Girifalco, L. A. \& Lad, R. A. Energy of cohesion, compressibility, and the potential energy functions of the graphite system. J. Chem. Phys. 25, 693 (1956).

2. Benedict, L. X. et al. Microscopic determination of the interlayer binding energy in graphite. Chem. Phys. Lett. 286, 490 (1998).

3. Girifalco, L. A. \& Hodak, M. Van der Waals binding energies in graphitic structures. Phys. Rev. B 65, 125404 (2002).

4. Zacharia, R., Ulbricht, H. \& Hertel, T. Interlayer cohesive energy of graphite from thermal desorption of polyaromatic hydrocarbons. Phys. Rev. B 69, 155406 (2004)

5. Hasegawa, M. \& Nishidate, K. Semiempirical approach to the energetics of interlayer binding in graphite. Phys. Rev. B 70, 205431 (2004).

6. Chakarova-Käck, S. D., Schröder, E., Lundqvist, B. I. \& Langreth, D. C. Application of van der Waals density functional to an extended system: Adsorption of benzene and naphthalene on graphite. Phys. Rev. Lett. 96, 146107 (2006).

7. Haas, P., Tran, F. \& Blaha, P. Calculation of the lattice constant of solids with semilocal functionals. Phys. Rev. B 79, 085104 (2009).

8. Spanu, L., Sorella, S. \& Galli, G. Nature and strength of interlayer binding in graphite. Phys. Rev. Lett. 103, 196401 (2009).

9. Lebègue, S. et al. Cohesive properties and asymptotics of the dispersion interaction in graphite by the random phase approximation. Phys. Rev. Lett. 105 196401 (2010)

10. Novoselov, K. S. et al. Two-dimensional gas of massless Dirac fermions in graphene. Nature 438, 197 (2005).

11. Taychatanapat, T., Watanabe, K., Taniguchi, T. \& Jarillo-Herrero, P. Quantum Hall effect and Landau-level crossing of Dirac fermions in trilayer graphene. Nat. Phys. 7, 621 (2011)

12. Kumar, A. et al. Integer quantum Hall effect in trilayer graphene. Phys. Rev. Lett. 107, 126806 (2011).

13. Bernal, J. D. Proc. R. Soc. Lond. 106, 749 (1924).

14. Haering, R. Band structure of rhombohedral graphite. Can. J. Phys. 36, 352 (1958)

15. Tan, P. et al. The shear mode of multilayer graphene. Nat. Mater. 11, 294 (2012).

16. Chen, N.-X. Möbius Inversion In Physics (World Scientific, Singapore, 2010)

17. Zhao, H., Chen, N.-X. \& Long, Y. Interfacial potentials for $\mathrm{Al} / \mathrm{SiC}$ (111). J. Phys.: Condens. Matter 21, 225002 (2009).

18. Tian, F.-Y., Chen, N.-X., Shen, J. \& Vitos, L. A novel potential: the interlayer potential for the fcc (111) plane family. J. Phys.: Condens. Matter 24, 045001 (2012).

19. Cançado, L. G. et al. Measuring the degree of stacking order in graphite by Raman spectroscopy. Carbon 46, 272 (2008).

20. Zhang, W. et al. Molecular adsorption induces the transformation of rhombohedral- to Bernal-stacking order in trilayer graphene. Nat. Commun. 4, (2013).

21. Huang, M. et al. Phonon softening and crystallographic orientation of strained graphene studied by Raman spectroscopy. Proc. Natl. Acad. Sci. 106, 7304 (2009).

22. Gould, T., Simpkins, K. \& Dobson, J. F. Theoretical and semiempirical correction to the long-range dispersion power law of stretched graphite. Phys. Rev. B 77, 165134 (2008).
23. Lipson, H. \& Stokes, A. The structure of graphite. Proc. R. Soc. Lond. 101 (1942)

24. Nicklow, R., Wakabayashi, N. \& Smith, H. Lattice dynamics of pyrolytic graphite. Phys. Rev. B 5, 4951 (1972)

25. Ooi, N., Rairkar, A. \& Adams, J. B. Density functional study of graphite bulk and surface properties. Carbon 44, 231 (2006).

26. Perdew, J. P., Burke, K. \& Ernzerhof, M. Generalized gradient approximation made simple. Phys. Rev. Lett. 77, 3865 (1996).

27. Grimme, S. Semiempirical GGA-type density functional constructed with a longrange dispersion correction. J. Comput. Chem. 27, 1787 (2006).

28. Dion, M., Rydberg, H., Schröder, E., Langreth, D. C. \& Lundqvist, B. I. Van der Waals density functional for general geometries. Phys. Rev. Lett. 92, 24640 (2004).

29. Lee, K., Murray, E. D., Kong, L., Lundqvist, B. I. \& Langreth, D. C. Higheraccuracy van der Waals density functional. Phys. Rev. B 82, 081101 (2010).

30. Kresse, G. \& Joubert, D. From ultrasoft pseudopotentials to the projector augmented-wave method. Phys. Rev. B 59, 1758 (1999).

31. Blöchl, P. E. Projector augmented-wave method. Phys. Rev. B 50, 17953 (1994).

32. Moruzzi, V. L., Janak, J. F. \& Schwarz, K. Calculated thermal properties of metals. Phys. Rev. B 37, 790 (1988).

33. Baskin, Y. \& Meyer, L. Lattice constants of graphite at low temperatures. Phys. Rev. 100, 544 (1955).

34. Gauster, W. B. \& Fritz, I. J. Pressure and temperature dependences of the elastic constants of compression-annealed pyrolytic graphite. J. Appl. Phys. 45, 3309 (1974).

35. Bosak, A., Krisch, M., Mohr, M., Maultzsch, J. \& Thomsen, C. Elasticity of singlecrystalline graphite: Inelastic X-ray scattering study. Phys. Rev. B 75, 153408 (2007).

36. Wada, N., Clarke, R. \& Solin, S. A. X-ray compressibility measurements of the graphite intercalates KC8 and KC24. Solid State Commun. 35, 675 (1980).

37. Liu, Z. et al. Interlayer binding energy of graphite: A mesoscopic determination from deformation. Phys. Rev. B 85, 205418 (2012).

\section{Acknowledgements}

We acknowledge the calculation resources provided by Research Computing Services (RCS) at USIT, UiO, and computational resources via the Swedish National Infrastructure for Computing (SNIC), including Center for Parallel Computers (PDC), Royal Inst of Technology, National Supercomputer Centre (NSC), Linköping University, and High Performance Computing Center North (HPC2N), Umeå University. This work was supported by the National Basic Research Development Program of China (Grant No. 2011CB606401), National Natural Science Foundation of China (Grant No. 11074139), and the Ministry of Science and Technology of China (Grants No. 2011CB606405 and No. 2011CB921901).

\section{Author contributions}

X.C. and F.T. have the same contribution to this work. N.-X.C. led the research and developed the theoretical model. Ab initio calculation was performed by C.P., X.C. and F.T. conducted the post-analysis separately and in parallel. The corresponding author is N.-X.C and he co-wrote the manuscript with F.T., X.C. and W.D. All authors contributed to discussions.

\section{Additional information}

Competing financial interests: The authors declare no competing financial interests.

How to cite this article: Chen, X.B., Tian, F.Y., Persson, C., Duan, W.H. \& Chen, N.-X. Interlayer interactions in graphites. Sci. Rep. 3, 3046; DOI:10.1038/srep03046 (2013)

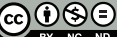

This work is licensed under a Creative Commons Attribution-

NonCommercial-NoDerivs 3.0 Unported license. To view a copy of this license, visit http://creativecommons.org/licenses/by-nc-nd/3.0 